\title{
Discovery of slow X-ray pulsations in the high-mass $X$-ray binary 4U 2206+54
}

\author{
P. Reig ${ }^{1,2}$, J. M. Torrejón ${ }^{3,4}$, I. Negueruela ${ }^{3}$, P. Blay ${ }^{5}$, M. Ribó ${ }^{6}$, and J. Wilms ${ }^{7}$
}

\author{
1 IESL, Foundation for Reseach and Technology-Hellas, 71110 Heraklion, Greece \\ 2 Physics Department, University of Crete, 71003 Heraklion, Greece \\ e-mail: pau@physics.uoc.gr \\ Departamento de Física, Ingeniería de Sistemas y Teoría de la Señal, Universidad de Alicante, 03080 Alicante, Spain \\ 4 Kavli Institute for Astrophysics and Space Research, Massachusetts Institute of Technology, Cambridge MA 02139, USA \\ 5 Institut de Ciencia dels Materials, Universitat de Valencia, 46071 Paterna-Valencia, Spain \\ ${ }^{6}$ Departament d'Astronomia i Meteorologia, Universitat de Barcelona, Martí i Franquès 1, 08028 Barcelona, Spain \\ 7 Dr. Karl Remeis-Observatory, University of Erlangen-Nuremberg, Sternwartstrasse 7, 96049 Bamberg, Germany
}

Received 10 September 2008 / Accepted 3 December 2008

\section{ABSTRACT}

\begin{abstract}
Context. The source $4 \mathrm{U} 2206+54$ is one of the most enigmatic high-mass X-ray binaries. In spite of intensive searches, X-ray pulsations have not been detected in the time range $10^{-3}-10^{3} \mathrm{~s}$. A cyclotron line at $\sim 30 \mathrm{keV}$ has been suggested by various authors but never detected with significance. The stellar wind of the optical companion is abnormally slow. The orbital period, initially reported to be 9.6 days, disappeared and a new periodicity of 19.25 days emerged.

Aims. The main objective of our RXTE monitoring of $4 \mathrm{U} 2206+54$ is to study the X-ray orbital variability of the spectral and timing parameters. The new long and uninterrupted RXTE observations allow us to search for long $(\sim 1 \mathrm{~h})$ pulsations for the first time. Methods. We divided the $\sim 7$-day observation into five intervals and obtained time-averaged energy spectra and power spectral density for each observation interval. We also searched for pulsations using various algorithms.

Results. We have discovered 5560-s pulsations in the light curve of 4U 2206+54. Initially detected in RXTE data, these pulsations are also present in INTEGRAL and EXOSAT observations. The average X-ray luminosity in the energy range $2-10 \mathrm{keV}$ is $1.5 \times 10^{35} \mathrm{erg} \mathrm{s}^{-1}$ with a ratio $F_{\max } / F_{\min } \approx 5$. This ratio implies an eccentricity of $\sim 0.4$, somewhat higher than previously suggested. The power spectrum is dominated by red noise that can be fitted with a single power law whose index and strength decrease with $\mathrm{X}$-ray flux. The source also shows a soft excess at low energies. If the soft excess is modelled with a blackbody component, then the size and temperature of the emitting region agrees with its interpretation in terms of a hot spot on the neutron star surface.

Conclusions. The discovery of X-ray pulsations in 4U 2206+54 confirms the neutron star nature of the compact companion and definitively rules out the presence of a black hole. The source displays variability on time scales of days, presumably due to changes in the mass accretion rate as the neutron star moves around the optical companion in a moderately eccentric orbit. If current models for the spin evolution in X-ray pulsars are correct, then the magnetic field of $4 \mathrm{U} 2206+54$ at birth must have been $B \gtrsim 10^{14} \mathrm{G}$.
\end{abstract}

Key words. stars: early-type - stars: emission-line, Be - stars: binaries: close - X-rays: binaries - stars: pulsars: general

\section{Introduction}

The X-ray source $4 \mathrm{U} 2206+54$ is one of the most enigmatic high-mass X-ray binaries (HMXB). Although it has been studied by numerous ground- and space-based observatories, many questions about the nature of its variability patterns remain unanswered. In the optical and $U V$ bands, the emitting spectrum is complex (Negueruela \& Reig 2001) and far from that of a $\mathrm{Be}$ star. Initially classified as a B0-2 star (Steiner et al. 1984), the optical counterpart defies any standard spectral analysis. The absence of any obvious long-term trends in the evolution of the $\mathrm{H} \alpha$ line spectral parameters and the lack of any correlation between the $\mathrm{H} \alpha$ line equivalent width and infrared magnitudes and colours rules out a Be classification. However, the strength and shape of the $\mathrm{H} \alpha$ line do not resemble those seen in supergiant stars either. Although the presence of an $09.5 \mathrm{~V}$ star is virtually secured, the He abundance is abnormally strong (Blay et al. 2006).

Without a circumstellar disc around the donor, the material needed for accretion and production of X-rays must come from the stellar wind. However, the expected X-ray luminosity using the typical wind velocities of an $09.5 \mathrm{~V}$ star is about two orders of magnitude lower than the observed X-ray luminosity $L_{\mathrm{X}} \sim 10^{35} \mathrm{erg} \mathrm{s}^{-1}$. The distance to $4 \mathrm{U} 2206+54$ is estimated to be $2.6 \mathrm{kpc}$ (Blay et al. 2006). Ribó et al. (2006) found that the wind terminal velocity of $4 \mathrm{U} 2206+54$ is abnormally slow $\left(\sim 350 \mathrm{~km} \mathrm{~s}^{-1}\right)$. With such a low velocity, an eccentric orbit and using the Bondi-Hoyle formalism it is possible to reproduce the average X-ray luminosity of the system as well as the orbital variability seen in the RXTE/ASM light curve between 1996 and 2005. 4U $2206+54$ is the only permanent wind-fed HMXB with a main-sequence donor (Ribó et al. 2006).

Equally enigmatic is the nature of the compact companion, which has not been settled beyond doubt. Although a neutron star is the most likely scenario, the low X-ray luminosity, compared to other persistent HMXB, has been used as an argument to suggest the presence of a white dwarf (Saraswat \& Apparao 1992; Corbet \& Peele 2001), while the lack of pulsations and the similarities with LS 5039 do not allow us to rule out a black hole companion (Negueruela \& Reig 2001) - the most recent results 
show, however, that LS 5039 is probably a non-accreting neutron star (Ribó et al. 2008).

Broad-band X-ray observations favour the presence of a neutron star (Torrejón et al. 2004). When modelled with Comptonization models, the resulting seed photons have $k T>$ $1 \mathrm{keV}$, while the bolometric luminosity is low $\left(L_{\mathrm{X}} \approx\right.$ $10^{35} \mathrm{erg} \mathrm{s}^{-1}$ ). The only way to reconcile these two results is by invoking a small emitting area $(<2 \mathrm{~km})$, like that of a polar cap in a neutron star (Masetti et al. 2004), also ruling out the presence of an accretion disc (Torrejón et al. 2004). The presence of a magnetic field with $B \sim 2 \times 10^{12} \mathrm{G}$ is implied by the possible detection of a cyclotron resonance scattering feature at $\sim 30 \mathrm{keV}$. Although the statistical significance of this feature is only marginal, it has been observed by three different observatories, namely RXTE, BeppoSAX and INTEGRAL (Torrejón et al. 2004; Masetti et al. 2004; Blay et al. 2005).

Another debated question is the orbital period of the system. Corbet \& Peele (2001) reported an orbital period of 9.6 days. This value was based on observations made with the RXTE ASM instrument over 5 years. This orbital period was refined to 9.5591 days by Ribó et al. (2006) using 9 years worth of data of the same instrument. However, Corbet et al. (2007) could not find the 9.6-day modulation in the light curves of SWIFT BAT and RXTE ASM for observations carried out from 2004 to 2005. Instead, they found a strong modulation at $19.25 \mathrm{~d}$, that is, consistent with twice the 9.6-day period (or $19.12 \mathrm{~d}$ ).

In this paper we investigate the X-ray variability of $4 \mathrm{U} 2206+54$ on time scales of hours to days. The main objective is to search for $\sim 1$-h pulsations and study possible spectral and timing orbital variability.

\section{Observations}

4U 2206+54 was observed by the Rossi X-ray Timing Explorer (RXTE) during the interval 15-22 May 2007 (JD $2454235.1628-2454242.1991$ ) for a total on-source time of $136.8 \mathrm{ks}$. Observation time with RXTE was awarded in response to the A11 call for proposals and corresponds to the $R X T E$ proposal P92068. Light curves, spectra and response matrices were extracted using the tools available in the RXTE subpackage of version v.6.4 of the FTOOLS software package.

$R X T E$ is designed to facilitate the study of time variability in the emission of X-ray sources with moderate spectral resolution. The pay-load consists of a Proportional Counter Array (PCA), which is sensitive to X-rays in the $2-60 \mathrm{keV}$ energy range and has a total effective area of $6500 \mathrm{~cm}^{2}$, shared by five PCU or proportional counter units (Jahoda et al. 1996). PCA data can be collected and telemetered to the ground in many different ways depending on the intensity of the source and the spectral and timing resolution desired. In this work we used the two standard modes: Standardl provides 0.125 -s resolution and no energy resolution; in the Standard2 configuration data are accumulated every $16 \mathrm{~s}$ in 129 channels. The High Energy X-ray Timing Experiment (HEXTE) comprises two independent clusters of detectors with a total collecting area of $1400 \mathrm{~cm}^{2}$, covering the energy range $15-250 \mathrm{keV}$. The spectral resolution is $\sim 15 \%$ at $60 \mathrm{keV}$ energy range. During normal operation, the two clusters rock on and off target to collect background data (Rothschild et al. 1998). Due to malfunction of the rocking mechanism, cluster A was left permanently on the on-source position for the entire duration of the observations. Thus, no background light curve could be obtained. Data presented in this work correspond to cluster B only. The third instrument on board RXTE is the All-Sky Monitor (ASM), which produce daily flux averages in
Table 1. Log of the RXTE observations.

\begin{tabular}{ccccc}
\hline \hline ObsID & MJD start-MJD stop & $\begin{array}{c}\text { On-source } \\
\text { time (s) }\end{array}$ & $\begin{array}{c}I_{\mathrm{X}}^{a} \\
\text { c s }^{-1}\end{array}$ & $\begin{array}{c}\text { Obs. } \\
\text { int. }\end{array}$ \\
\hline $01-01-05$ & $54235.1628-54235.1908$ & 1600 & 17.8 & $\mathrm{~A}$ \\
$01-01-02$ & $54235.8369-54236.1095$ & 23472 & 28.2 & $\mathrm{~A}$ \\
$01-01-03$ & $54236.8613-54237.1036$ & 20672 & 28.0 & $\mathrm{~B}$ \\
$01-01-04$ & $54237.8639-54238.0000$ & 11696 & 30.0 & $\mathrm{~B}$ \\
$01-02-00$ & $54238.0010-54238.0841$ & 7120 & 18.1 & $\mathrm{C}$ \\
$01-02-01$ & $54238.8408-54239.0647$ & 17328 & 8.8 & $\mathrm{C}$ \\
$01-02-01$ & $54239.0865-54239.1199$ & 2384 & 7.4 & $\mathrm{D}$ \\
$01-02-02$ & $54239.8821-54240.0452$ & 11328 & 6.6 & $\mathrm{D}$ \\
$01-02-02$ & $54240.0671-54240.1124$ & 2512 & 5.6 & $\mathrm{D}$ \\
$01-02-02$ & $54240.1365-54240.1799$ & 2256 & 3.2 & $\mathrm{D}$ \\
$01-02-03$ & $54240.8137-54241.0263$ & 13584 & 5.7 & $\mathrm{D}$ \\
$01-02-03$ & $54241.0482-54241.0930$ & 2528 & 7.9 & $\mathrm{E}$ \\
$01-02-05$ & $54241.1412-54241.1604$ & 400 & 6.9 & $\mathrm{E}$ \\
$01-02-040$ & $54241.7899-54241.9452$ & 9328 & 10.7 & $\mathrm{E}$ \\
$01-02-040$ & $54241.9545-54242.0069$ & 3776 & 7.3 & $\mathrm{E}$ \\
$01-02-040$ & $54242.0287-54242.0743$ & 2544 & 6.2 & $\mathrm{E}$ \\
$01-02-04$ & $54242.0982-54242.1408$ & 2256 & 5.1 & $\mathrm{E}$ \\
$01-02-04$ & $54242.1663-54242.1991$ & 1984 & 4.9 & $\mathrm{E}$ \\
\hline
\end{tabular}

${ }^{a}$ Background subtracted 4-30 keV, PCU2 only.

the energy range $1.3-12.1 \mathrm{keV}$ at $30 \mathrm{mCrab}$ sensitivity (Levine et al. 1996).

The log of the RXTE observations is shown in Table 1. Good time intervals were defined when the pointing of the satellite was stable $\left(<0.02^{\circ}\right.$ from the source $)$, the elevation above $8^{\circ}$ and far away from the South Atlantic Anomaly. Due to RXTE's lowEarth orbit, the data consist of a number of contiguous data intervals (typically $1 \mathrm{~h}$ long) interspersed with observational gaps produced by Earth occultations of the source and passages of the satellite through the South Atlantic Anomaly. In general, the visibility of a target depends on its position with respect to the plane of RXTE's orbit around the Earth. Objects that are within a critical angle from the normal to the orbit will be visible for longer periods of time. In fact, if the object is close enough to the normal then it would lie in the so-called Continuous viewing zone (CVZ). Since RXTE orbits relatively close to the Earth's equator, the regions of high visibility and the CVZ are close to the north and south pole of the Earth. 4U 2206+54 is at high ecliptic latitude (about 58 degrees), hence it has a relatively high visibility. The observations reported here are considerably longer than commonly reported for other sources. There are two long ( $\sim 6 \mathrm{~h})$ uninterrupted observations. These longer observations, together with the monitoring of $4 \mathrm{U} 2206+54$ over 7 days, allow us to give new insights into the properties of the X-ray emission in 4U 2206+54. In particular, they allow us to search for periodicities on timescales of $\sim 1 \mathrm{~h}$. To investigate potential orbital variability, the RXTE observations were divided into five intervals with roughly the same on-source time, $\sim 20-30 \mathrm{ks}$ (Fig. 1).

In addition to the RXTE pointing observations, we also analysed archived data from the INTEGRAL and EXOSAT missions. The International Gamma-ray Astrophysics Laboratory (INTEGRAL) observations were made between 1619 December 2006 during revolution 510. INTEGRAL carries two main gamma-ray instruments: the high angular resolution Imager (IBIS) and the high-energy resolution spectrometer (SPI). In our study we only used data from the upper detector system of IBIS, called the Soft Gamma-ray Imager (ISGRI). 


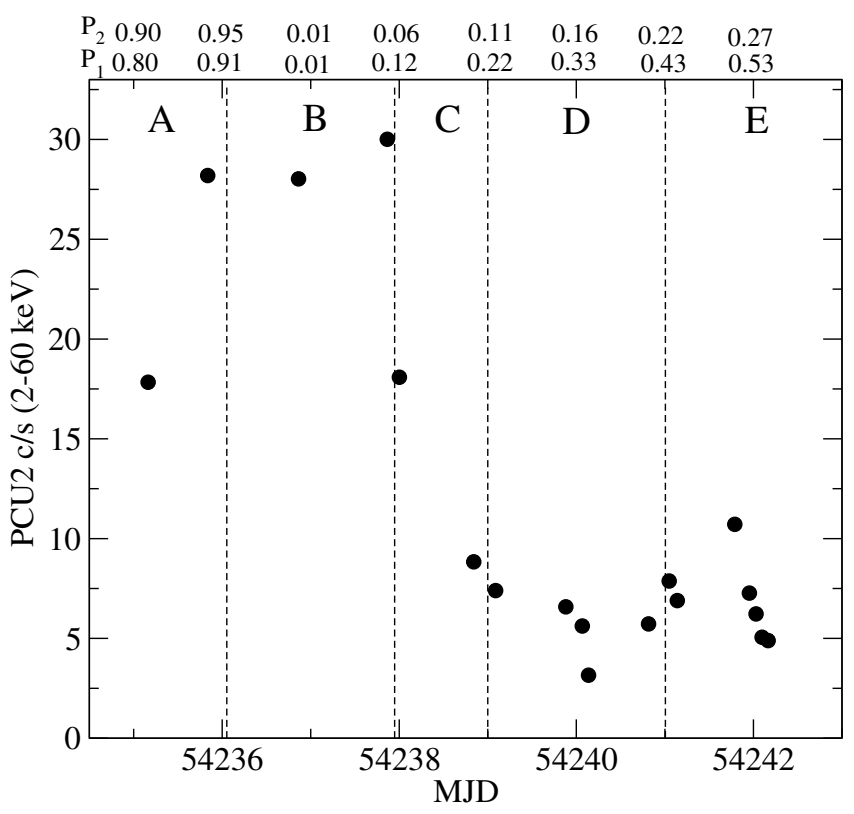

Fig. 1. Light curve of $4 \mathrm{U} 2206+54$ covering the entire interval of the RXTE PCA observations. The data points represent the average of each observation interval as given in Table 1. Errors are less than the size of the points. Dashed lines separate the different intervals for orbital variability analysis (see text). The numbers on top of the figure are the orbital phase for $P_{1}=9.56 \mathrm{~d}$ and $P_{2}=19.12 \mathrm{~d}$. Reference time for phase calculation is MJD 53567.74, which corresponds to maximum flux in the folded ASM light curve (Corbet et al. 2007).

ISGRI is sensitive to photons in the energy range $20 \mathrm{keV}$ to $1 \mathrm{MeV}$, has an angular resolution of $12^{\prime}$ and a source location accuracy that depends on the significance of the detection: for a source at the limit of detection the source location accuracy is about $4-5^{\prime}$ while for a strong source it can go down to about $0.5^{\prime}$ (Gros et al. 2003). The energy resolution is $7 \%$ at $100 \mathrm{keV}$ (Ubertini et al. 2003). 4U 2206+54 was detected in a total of 19 INTEGRAL science windows of around $\sim 2.3 \mathrm{ks}$ exposure each. In all these observations the satellite followed a $5 \times 5$ dithering pattern. We only analysed images in which the source fell in the fully coded field of view $\left(9^{\circ} \times 9^{\circ}\right)$. Data reduction was done with the Offline Scientific Analysis (OSA) software version 7 (Courvoisier et al. 2003).

The European Space Agency's X-ray Observatory, EXOSAT, was operational from May 1983 to April 1986. The pay-load consisted of 2 Wolter type I grazing incidence Low Energy (LE; 0.05-2 keV) Imaging Telescopes, a medium energy (ME) Proportional Counter and a gas scintillation (GS) Proportional Counter. The ME detectors covered the energy range $1-50 \mathrm{keV}$ and had a field of view of 45 arcmin and an effective area $1600 \mathrm{~cm}^{2}$ (Turner et al. 1981). For our study we retrieved the ME detector standard products available in the NASA's HEASARC archive. EXOSAT observed 4U 2206+54 with the Medium Energy (ME) proportional counter in three occasions: 8 August 1983, 7 December 1984 and 27 June 1985 for a total of $9.8 \mathrm{ks}, 9.3 \mathrm{ks}$ and $6.3 \mathrm{ks}$, respectively.

\section{Timing analysis}

We have performed a timing analysis on two time scales: a) minutes to hours to search for slow pulsations; and b) days to investigate orbital variability. Figure 1 shows the entire $R X T E / P C A$ light curve. Each point represents the average of one observation

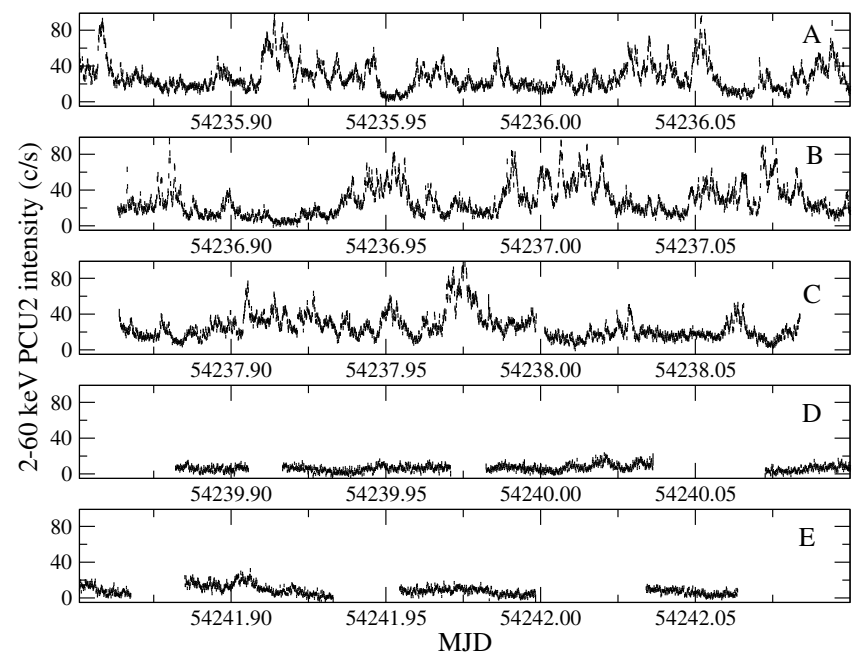

Fig. 2. Portion of $\sim 6 \mathrm{~h}$ of the light curves of $4 \mathrm{U} 2206+54$ corresponding to each one of the observation intervals. The axis scales were left the same in all panels to allow easy comparison of the variability amplitude. The bin size is $10 \mathrm{~s}$. Intervals $\mathrm{C}$ and $\mathrm{D}$ are more affected by observational gaps.

interval (see Table 1). On short time scales the X-ray light curve of $4 \mathrm{U} 2206+54$ is dominated by erratic flaring, with changes in the X-ray intensity by a factor 3-5 over a few minutes (Fig. 2). Despite the different wind parameters and optical companion believed to be present in 4U 2206+54 (Blay et al. 2006; Ribó et al. 2006), the light curves are very similar to those of $X$-ray sources in which a neutron star is fed by direct accretion from the wind of a supergiant, such as Vela X-1. This supports the idea that accretion in this source is not mediated by an accretion disc, but proceeds directly from the wind. Figure 2 shows a portion of the light curve for each interval. The $Y$-axis spans the same intensity range in all panels to show the large variability of $4 \mathrm{U} 2206+54$ on short timescales.

Figure 3 shows a colour-intensity diagram where three hardness ratios are plotted as a function of $4-30 \mathrm{keV}$ PCU2 count rate. Each point represents an average over 512 s. The source becomes harder as the count rate increases. A fit to a constant function does not give good fits to the top two panels, with reduced $\chi_{\mathrm{r}}^{2}$ of 8.5 and 5.1, indicating that the variation is statistically significant. In contrast, the data of the bottom panel give $\chi_{\mathrm{r}}^{2}=1.4$. The correlation coefficients to a linear regression fits in the three panels are, from top to bottom, 0.6, 0.7 and -0.2 .

\subsection{Search for long-term pulsations}

In spite of intensive searches, X-ray pulsations have not been detected in $4 \mathrm{U} 2206+54$. Searches for X-ray coherent emission have been performed with EXOSAT (Corbet \& Peele 2001, but see Saraswat \& Apparao 1992), RXTE (Negueruela \& Reig 2001; Torrejón et al. 2004; Corbet et al. 2007), BeppoSAX (Masetti et al. 2004; Torrejón et al. 2004) and INTEGRAL (Blay et al. 2005). These studies demonstrate the lack of X-ray pulsations on timescales from $\sim 1 \mathrm{~ms}$ to $\sim 1 \mathrm{~h}$. The possible detection of 392-s pulsations reported by Saraswat \& Apparao (1992) has never been confirmed. Corbet \& Peele (2001) reanalysed the EXOSAT observations used by Saraswat \& Apparao (1992) and did not find any evidence of any pulsations longer than $2 \mathrm{~s}$. They argue that Saraswat \& Apparao (1992) used a procedure not well suited to search for coherent periodic pulses. 


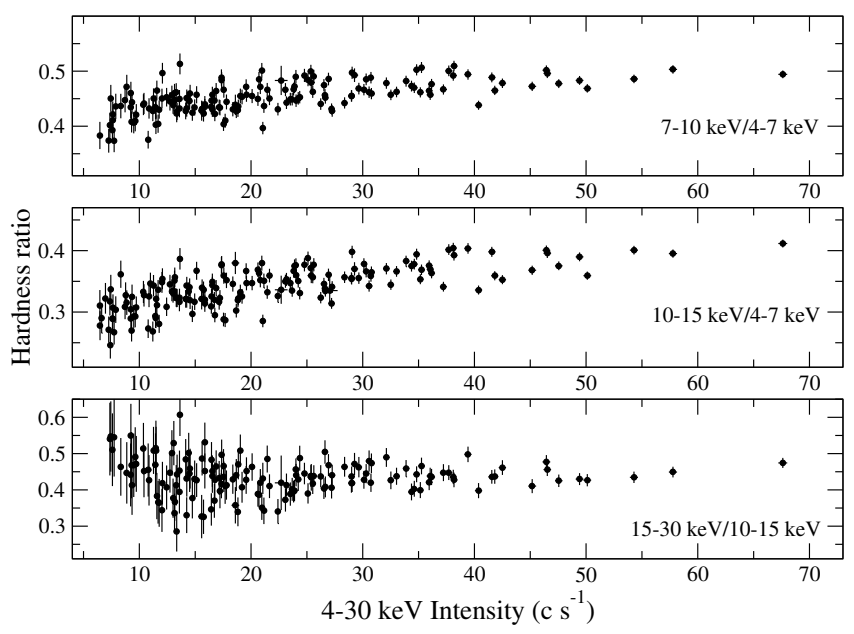

Fig. 3. Hardness-intensity diagram of $4 \mathrm{U} 2206+54$ for various X-ray bands. Count rates correspond to PCU2. Bin size is $512 \mathrm{~s}$.

The only timescales that have not been investigated in detail due to either technical constraints (low-Earth orbits) or lack of long uninterrupted observations is from $\sim 1 \mathrm{~h}$ to $\sim 1 \mathrm{day}$. The long coverage of our RXTE observations allows us to investigate the higher frequency part of this unexplored interval.

We searched for periodic signals in the light curves using various standard techniques. Initially we applied an FFT to the two longest observation intervals (see Table 1). However, in order to be able to use the entire data set, techniques suitable for handling missing data (gaps) in the light curves are needed. Thus we employed PERIOD04 (Lenz \& Breger 2005), the Phase Dispersion Minimisation (PDM, Stellingwerf 1978) and the CLEAN algorithm (Roberts et al. 1987). A periodic signal around $\sim 5560 \mathrm{~s}$ is clearly detected with all methods. The PDM and CLEAN periodograms obtained between 100 and $10000 \mathrm{~s}$ for the $R X T E / \mathrm{PCA}$ light curve binned at $16 \mathrm{~s}$ intervals are shown in the top panel of Fig. 4. In the case of PDM there is a strong beating with $\sim 400 \mathrm{~s}$ separation, and two equally significant minima occur at $5555 \pm 10 \mathrm{~s}$ and $5608 \pm 10 \mathrm{~s}$. In the case of CLEAN there is a clear maximum only at $5559 \pm 3 \mathrm{~s}$.

To further study the periodic signal detected with the whole data set, we have analysed the $R X T E / \mathrm{PCA}$ light curves of intervals $\mathrm{A}+\mathrm{B}$ and intervals $\mathrm{C}+\mathrm{D}+\mathrm{E}$ separately, corresponding to a high and low flux states of the source on an orbital timescale (Fig. 1). The results show that the periodic signal is detected in both data sets, clearly indicating that it is independent of the X-ray flux of the source. In particular, for A+B PDM and CLEAN provide signals at $5550 \pm 50 \mathrm{~s}$ and $5562 \pm 3 \mathrm{~s}$, respectively. For $\mathrm{C}+\mathrm{D}+\mathrm{E}$ we obtain $5560 \pm 30 \mathrm{~s}$ and $5559 \pm 3 \mathrm{~s}$, respectively. The probability that the detected period is due to random fluctuations is less than $10^{-8}$, according to the statistical properties of the PDM algorithm (Stellingwerf 1978).

We note that a strong signal is also detected around $815000 \pm 15000 \mathrm{~s}(9.4 \pm 0.2 \mathrm{~d})$ with the CLEAN algorithm in the RXTE/PCA light curve. Although the available database is limited to just $7.0 \mathrm{~d}$, it is interesting to note that a clear hint of the possible $9.6 \mathrm{~d}$ (or of a part of the $19.25 \mathrm{~d}$ ) orbital period appears in the $R X T E / \mathrm{PCA}$ data set of $4 \mathrm{U} 2206+54$. The probability that there is not such periodicity or that the real period is different to $815000 \mathrm{~s}$ is less than 0.01 .

The value of $5560 \mathrm{~s}$ is very close to RXTE's orbital period RXTE follows a low-Earth circular orbit at an altitude of $580 \mathrm{~km}$, corresponding to an orbital period of about $90 \mathrm{~min}$. Note that the
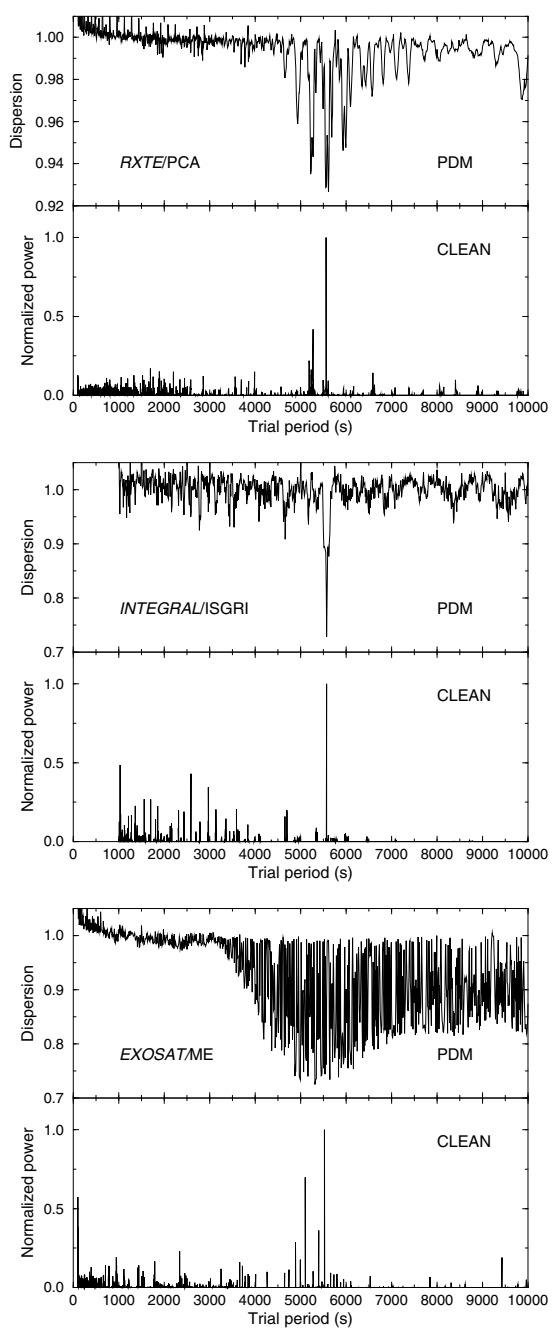

Fig. 4. Periodograms of the entire light curves of 4U 2206+54 for three different instruments obtained by using the PDM and CLEAN algorithms. A periodic signal is clearly detected at $\sim 5560 \mathrm{~s}$.

$R X T E$ 's orbital period is continuously changing due to perturbations by the Earth and Moon and atmospheric drag. At the time of the observations the orbital period laid in the range 5667.3$5669.1 \mathrm{~s}$. In order to rule out an instrumental origin of the modulation, we analysed archived data from the INTEGRAL/ISGRI and EXOSAT/ME missions and instruments. The orbit of these two observatories is highly eccentric with a period of $72 \mathrm{~h}$ and $90 \mathrm{~h}$, respectively. The time bin was $500 \mathrm{~s}$ for the ISGRI light curve and $30 \mathrm{~s}$ for the ME light curve.

The timing analysis on the INTEGRAL (20-40 keV) and EXOSAT (0.8-9 keV) light curves gives periodic signals at $5570 \pm 20 \mathrm{~s}$ and $5525 \pm 30 \mathrm{~s}$, respectively (Fig. 4), which clearly confirms the presence of the 1.54-h periodic signal found with the $R X T E / P C A$ data.

Figure 5 shows the pulse profile corresponding to each satellite. The pulsed fraction is $\sim 50 \%$ for the RXTE and INTEGRAL light curves and $35 \%$ for the EXOSAT data.

HEXTE data were not used in the periodicity analysis because of the problems with cluster A (see Sect. 2) and the low $\mathrm{S} / \mathrm{N}$ of the cluster B light curves. Also, note that the energy range covered by HEXTE is partially covered by ISGRI/INTEGRAL. 


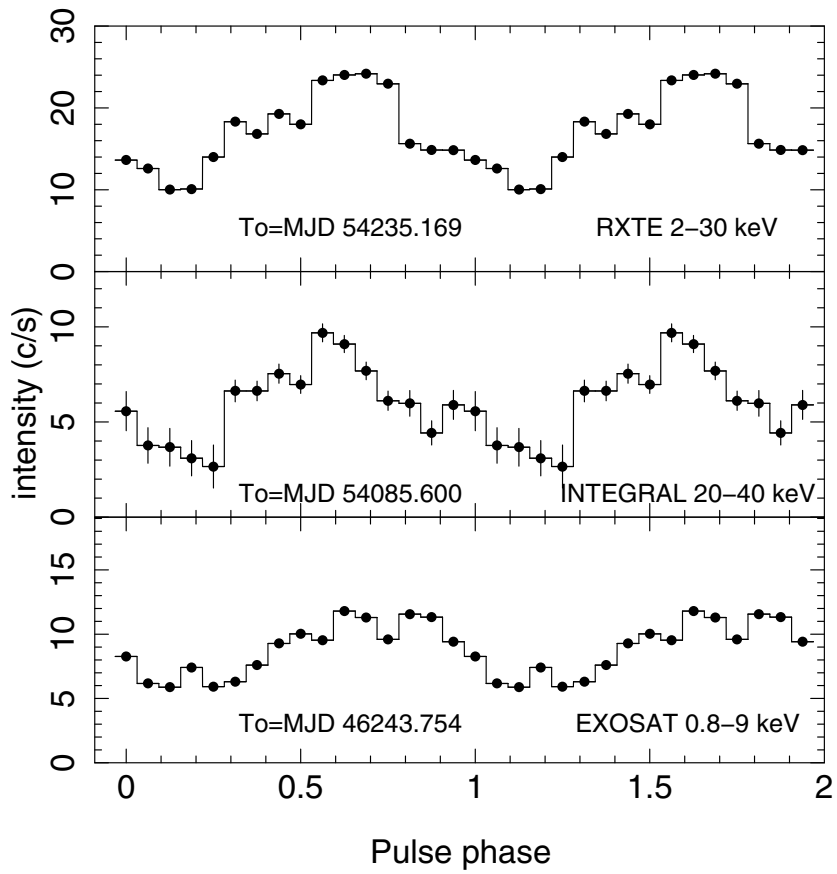

Fig. 5. Pulse profiles obtained from the light curves of RXTE/PCA, INTEGRAL/ISGRI, and EXOSAT/ME folded on to the period $P_{\text {pulse }}=$ 5559 s. Two pulse periods are shown for clarity.

\subsection{Orbital variability}

To investigate the longer-term variability, the observations were divided into five intervals with roughly the same on-source time. The average intensity decreased throughout the time covered by the observations as can be seen in Fig. 1. The numbers on top of this figure are the orbital phase assuming $P_{1}=9.56 \mathrm{~d}$ and twice that period $P_{2}=19.12$ d (Corbet et al. 2007). The reference time is the epoch of maximum flux as given by the ASM, MJD 53567.74 (Corbet et al. 2007). The PCA light curve shows maximum flux at phase $\sim 0$, in agreement with the modulation of the folded ASM light curve (Corbet et al. 2007). There is another less significant peak at about phase 0.5 , if $P_{\text {orb }}=9.56 \mathrm{~d}$. If the orbital period were $P_{\text {orb }}=19.12 \mathrm{~d}$, then we would expect to see the maximum flux around phase 0.125 (Corbet et al. 2007), which is not seen.

In order to investigate the evolution of the power spectral parameters of the noise components as the source moves around the orbit, we produced one power spectrum for each interval. The power spectra were obtained by dividing the 2-20 keV PCA $2^{-7}$-s binned light curve of the entire observation into 1024-s segments and an FFT obtained for each segment. The contribution by the photon counting noise was computed and subtracted from each power spectrum. The power spectra were normalized such that their integral gives the squared fractional rms variability (Belloni \& Hasinger 1990; Miyamoto et al. 1991). The power spectra of the same interval were averaged to produce the final power spectrum. A logarithmic rebinning was also applied to reduce the noise at high frequencies. Figure 6 shows the power spectra for the five intervals. The results of the fitting procedure are shown in Table 2.

A single power-law component provides good fits for all intervals. The power-law index and the fractional $\mathrm{rms}$ amplitude change with X-ray intensity. The high-intensity intervals (A and B) show a steeper power spectrum and a larger amplitude of variability. The low-intensity intervals (D and E) display a flatter
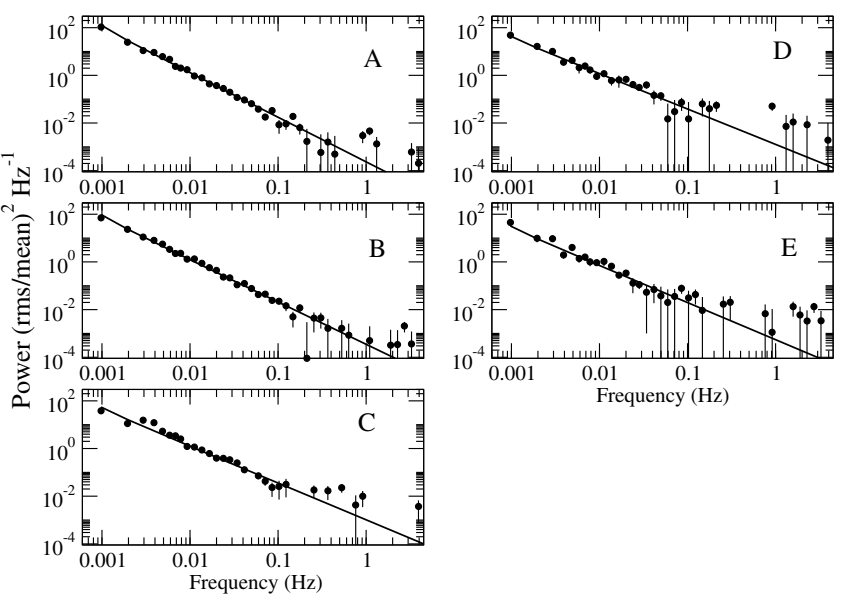

Fig. 6. Power spectra of 4 U $2206+54$ at different instances of the observations. See Table 2 for a definition of the intervals.

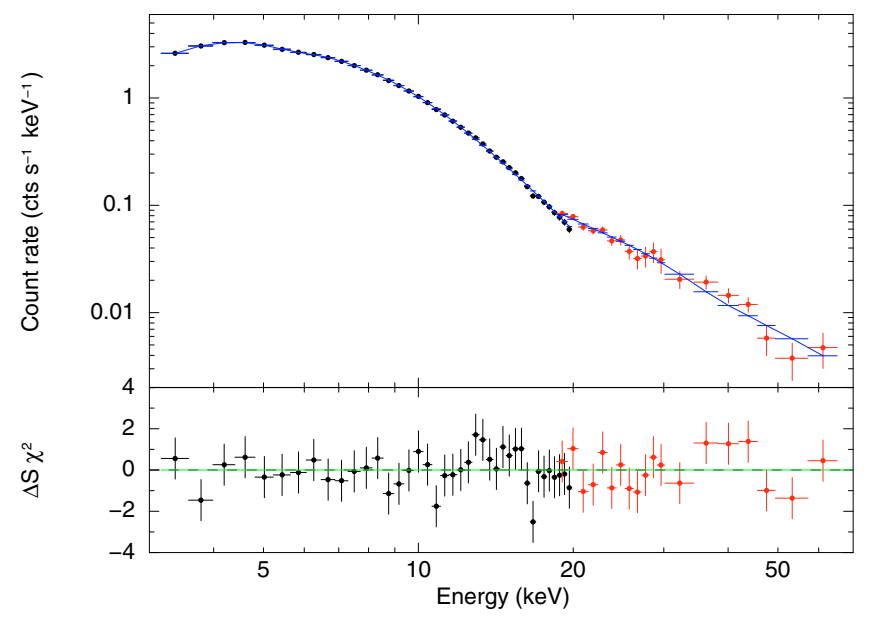

Fig. 7. X-ray spectrum in the energy range $3-70 \mathrm{keV}$ obtained from PCA and HEXTE and fitted with a thermal Comptonization model plus a black body at low energies (continuous line). Data to model residuals, in units of sigmas, are depicted in the lower panel.

power law and are less variable. Interval $\mathrm{C}$ shows intermediate values between these two cases.

\section{Spectral analysis}

In this section we present the results of the energy spectral analysis. We first present the PCA+HEXTE time-average spectrum for the entire observation. Then, we search for orbital variability in the spectral parameters.

\subsection{Time-averaged spectrum}

The 3-30 keV spectrum of $4 \mathrm{U} 2206+54$ is well described by an absorbed, $N_{\mathrm{H}}=1.3 \pm 0.5 \times 10^{22} \mathrm{~cm}^{-2}$, power law with photon index $1.3 \pm 0.1$ modified by a cutoff at $6.3 \pm 0.4 \mathrm{keV}$ and folding energy $14 \pm 2 \mathrm{keV}$, in agreement with those found by Negueruela \& Reig (2001). However, as shown in Torrejón et al. (2004), this model does not describe properly the spectrum when data beyond $30 \mathrm{keV}$ are taken into account $\left(\chi_{v}^{2}=1.44\right.$ for 53 d.o.f.). The joined time-average PCA+HEXTE spectrum (Fig. 7) is, overall, well described by thermal Comptonization (comptt in XSPEC notation). This model requires further a blackbody emission (bb) component to account for an excess at low energies. The same 
Table 2. Results of the power-law fits to the power spectra.

\begin{tabular}{cccccccc}
\hline \hline & MJD & $\begin{array}{c}I_{\mathrm{X}}^{a} \\
\mathrm{c} / \mathrm{s}\end{array}$ & $\begin{array}{c}\text { Orbital } \\
\text { phase }^{b}\end{array}$ & $\begin{array}{c}\text { Number } \\
\text { of PSD }\end{array}$ & $\Gamma$ & $\begin{array}{c}\text { rms (\%) } \\
(0.001-1 \mathrm{~Hz})\end{array}$ & $\chi_{\mathrm{r}}^{2} /$ d.o.f. \\
\hline $\mathrm{A}$ & $54235.16-54236.11$ & 29 & $0.87 / 0.93$ & 21 & $1.87 \pm 0.04$ & 37 & $1.3 / 44$ \\
$\mathrm{~B}$ & $54236.86-54238.00$ & 27 & $0.06 / 0.03$ & 26 & $1.77 \pm .04$ & 34 & $1.1 / 44$ \\
$\mathrm{C}$ & $54238.00-54239.06$ & 12 & $0.17 / 0.09$ & 19 & $1.55 \pm 0.05$ & 30 & $1.9 / 43$ \\
$\mathrm{D}$ & $54239.09-54241.03$ & 6 & $0.33 / 0.17$ & 24 & $1.5_{-0.13}^{+0.09}$ & 28 & $1.3 / 44$ \\
$\mathrm{E}$ & $54241.05-54242.20$ & 9 & $0.50 / 0.25$ & 15 & $1.5 \pm 0.1$ & 23 & $1.2 / 44$ \\
\hline
\end{tabular}

${ }^{a}$ Background subtracted 2-60 keV, PCU2 only; ${ }^{b}$ at mid-point for $P_{\text {orb }}=9.56 \mathrm{~d}$ and $P_{\text {orb }}=19.12 \mathrm{~d}$, respectively.

is true when dynamical Comptonization (bmc) replaces thermal Comptonization. The parameters for these two composite models are presented in Table $3 . k T_{\mathrm{bb}}$ is the temperature of the blackbody component that accounts for the soft excess; $k T_{0}$ and $k T_{\text {col }}$ are the temperatures of the seed photons, which are subsequently Comptonized by the high temperature plasma, in the thermal and dynamical Comptonization models respectively; $k T_{\mathrm{e}}$ and $\tau$ are the temperature and optical depth of the high temperature plasma, in the thermal Comptonization model; $\alpha$ is an spectral index related to the efficiency of the Comptonization process (the lower the value of $\alpha$, the more efficient is the Comptonization); $f$ is the illumination factor, i.e., the ratio of the number of multiply scattered photons to the total number of seed photons $(f \gg 1$ implies that the spectrum is fully Comptonized). No iron line is required. If the absorption column is let free to vary, then the best-fit value is higher than in previous observations. Fixing it to a lower value increases slightly the temperature of the blackbody but it is always around $0.5 \mathrm{keV}$ (for example fixing $N_{\mathrm{H}}$ to $0.3 \times 10^{22} \mathrm{~cm}^{-2}$, the pure interstellar value, gives $k T_{\mathrm{bb}}=0.56$ and sets the $\left.\chi_{v}^{2}=1\right)$. Since $R X T E$ cannot constrain the column density due to the lack of sensitiveness below $3 \mathrm{keV}$ we fixed $N_{\mathrm{H}}$ to the value reported from BeppoSAX observations, namely $N_{\mathrm{H}}=0.9 \times 10^{22} \mathrm{~cm}^{-2}$ (Masetti et al. 2004). Applying Eq. (1) in Torrejón et al. (2004) to the data in Table 3, we determine the radius of the soft photon source which would produce the observed luminosity ${ }^{1}$ to be $R_{W}=1.5 \mathrm{~km}$. This is only consistent with a hot spot on the neutron star surface.

The possible cyclotron line around $30 \mathrm{keV}$ reported by various authors (Torrejón et al. 2004; Masetti et al. 2004; Blay et al. $2005)$ is not seen in the present data set. We did not find significant evidence for the iron emission line at $\sim 6.4 \mathrm{keV}$ either. We estimate an upper limit on the equivalent width of a narrow line $(F W H M=0.1 \mathrm{keV})$ around $6.4 \mathrm{keV}$ of $25 \mathrm{eV}$ at $90 \%$ confidence level. The average $\mathrm{X}$-ray luminosity in the energy range $3-30 \mathrm{keV}$ is $2 \times 10^{35} \mathrm{erg} \mathrm{s}^{-1}$, assuming a distance of $2.6 \mathrm{kpc}$ (Blay et al. 2006).

\subsection{Pulse-phase spectroscopy}

In order to investigate how the X-ray spectrum changes as a function of the spin of the magnetized neutron star, the pulse profile corresponding to intervals $\mathrm{A}$ and $\mathrm{B}$ was divided into eight equally spaced bins and a spectrum in the energy range 3$20 \mathrm{keV}$ was obtained for each bin. After subtracting the underlying persistent emission, each spectrum was satisfactorily fitted with an absorbed power law. The column density was fixed to $1.3 \times 10^{22} \mathrm{~cm}^{-2}$, i.e., the value found in the time-averaged

1 This formula gives the proper factor for a circular spot of area $\pi R_{W}^{2}$. Of course, a sphere would require a radius $R^{\mathrm{sph}}=R^{\mathrm{spot}} / 2$ to produce the same $L_{\mathrm{X}}$ assuming isotropic emission.
Table 3. Parameters for the Comptonization models. Error bars are $90 \%$ confidence level.

\begin{tabular}{lclc}
\hline \hline \multicolumn{2}{c}{$\mathrm{bb}+\mathrm{comptt}$} & \multicolumn{2}{c}{$\mathrm{bb}+\mathrm{bmc}$} \\
\hline$N_{\mathrm{H}}^{a}$ & 0.9 & $N_{\mathrm{H}}^{a}$ & 0.9 \\
& & & \\
$k T_{\mathrm{bb}}(\mathrm{keV})$ & $0.55_{-0.07}^{+0.08}$ & $k T_{\mathrm{bb}}(\mathrm{keV})$ & $0.46_{-0.10}^{+0.08}$ \\
$N_{\text {orm }}$ & $\left(4.65_{-0.49}^{+1.25}\right) \times 10^{-4}$ & $\mathrm{Norm}_{\mathrm{bb}}$ & $\left(4.17_{-1.09}^{+5.72}\right) \times 10^{-4}$ \\
$F_{\mathrm{bb}}^{b}$ & 0.08 & $F_{\mathrm{bb}}^{b}$ & 0.04 \\
& & & \\
$k T_{0}(\mathrm{keV})$ & $1.30_{-0.05}^{+0.07}$ & $k T_{\mathrm{col}}(\mathrm{keV})$ & $1.30_{-0.05}^{+0.05}$ \\
$k T_{\mathrm{e}}(\mathrm{keV})$ & $35.20_{-1.26}^{+1.25}$ & $\alpha$ & $1.33_{-0.05}^{+0.84}$ \\
$\tau$ & $0.73_{-0.04}^{+0.03}$ & $f$ & $\mathrm{~N}^{-4}$ \\
$\mathrm{Norm}_{\mathrm{comptt}}$ & $\left(6.53_{-0.48}^{+1.05}\right) \times 10^{-4}$ & $\mathrm{Norm}_{\mathrm{bmc}}$ & $\left(1.68_{-0.01}^{+0.01}\right) \times 10^{-3}$ \\
$F_{\mathrm{comptt}}^{b}$ & 2.49 & $F_{\mathrm{bb}}^{b}$ & 2.52 \\
$\chi_{v}^{2}$ (d.o.f.) & $1.00(52)$ & $\chi_{v}^{2}$ (d.o.f.) & $0.94(53)$ \\
\hline
\end{tabular}

${ }^{a}$ Hydrogen column density in units of $10^{22} \mathrm{~cm}^{-2}$ (fixed); ${ }^{b} 3-60 \mathrm{keV}$ unabsorbed flux in units of $10^{-10} \mathrm{erg} \mathrm{s}^{-1} \mathrm{~cm}^{-2}$.
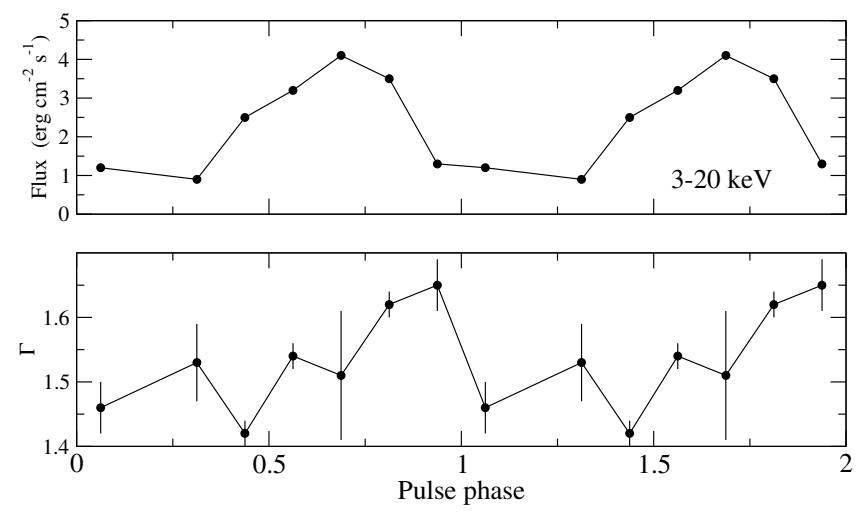

Fig. 8. X-ray flux and photon index as a function of the spin phase.

spectrum (Sect. 4.1), although the use of $N_{\mathrm{H}}=0.9 \times 10^{22} \mathrm{~cm}^{-2}$ did not change the results significantly. Figure 8 shows the variation of the 3-20 keV X-ray flux and the power-law photon index as a function of the pulse phase. Phase zero corresponds to MJD 54 235.169. Marginal evidence for variability in the powerlaw photon index is found. The spectrum becomes softer as the pulse phase increases, with the photon index reaching a maximum around phase 0.9 . The hardness of the spectrum does not seem to depend on the pulse flux, but the photon index appears to lag the pulse flux by about $1200 \mathrm{~s}$. However, the uncertainty in the photon index values is too large to draw a firm conclusion. 


\subsection{Orbital spectral variability}

To investigate possible orbital spectral variability, we obtained a time-averaged spectrum for each observation interval. The 3-30 keV band spectrum was fitted to an absorbed power law modified at high energies by an exponential cutoff. Given the high low-energy limit, the hydrogen column $N_{\mathrm{H}}$ is not well constrained. Although the observations that correspond to a lower flux have lower column densities, the value of $N_{\mathrm{H}}$ is consistent with no variability, within the errors. Thus, we also tried fits with a fixed $N_{\mathrm{H}}=0.9 \times 10^{22} \mathrm{~cm}^{-2}$ (lower part of Table 4). In either case, a variation in the power-law index is apparent. High-flux states show harder spectra.

\section{Discussion}

We have reported the analysis of the monitoring campaign of the high-mass X-ray binary $4 \mathrm{U} 2206+54$ that took place in May 2007 with $R X T E$. The observations span over $\sim 7$ days, which represent a large fraction of the 9.6-day period. The long and uninterrupted observations allow us for the first time to $i$ ) extend the search for X-ray pulsations to time scales of about one hour; ii) investigate the X-ray orbital variability; and iii) give new insights into its energy emission properties. In support of the results of these observations we also analysed data from INTEGRAL/ISGRI and EXOSAT/ME.

\subsection{X-ray pulsations}

The proximity of $4 \mathrm{U} 2206+54$ to $R X T E$ 's Continuous Viewing Zone allowed the acquisition of long and uninterrupted observations, and the discovery of slow X-ray pulsations $\left(P_{\text {spin }}=5559 \pm\right.$ $5 \mathrm{~s})$. With the discovery of X-ray pulsations we have solved one of the questions that remained unanswered, namely, the nature of the compact companion. The vast majority of HMXBs harbour $\mathrm{X}$-ray pulsars. These are believed to be young neutron stars with relatively strong magnetic fields $\left(B \sim 10^{12} \mathrm{G}\right)$. Among the handful of HMXBs not displaying X-ray pulsations, three show the typical characteristics of persistent black-hole systems. Of these, two are in the LMC and only one (Cyg X-1) in the Galaxy. The nature of the other three HMXBs in which pulsations have not been discovered in spite of intensive searches is under debate, although two of them may also contain black hole companions: LS I +61 303/2E 0236.6+6101 (Casares et al. 2005a, but see Dhawan et al. 2006) and LS 5039/RX J1826.2-1450 (Casares et al. 2005b, but see Ribó et al. 2008). If confirmed it would explain the lack of pulsations in these systems. Geometric effects (very low inclination or a very small angle between the spin and rotation axises) have been invoked to explain the lack of pulsations in 4U 1700-37, which might contain a massive neutron star (Clark et al. 2002; Abubekerov 2004). The lack of pulsations was more difficult to explain in $4 \mathrm{U} 2206+54$, as previous observations indicated that this source likely hosts a canonical neutron star accreting from the wind of a main-sequence earlytype star (Torrejón et al. 2004; Masetti et al. 2004; Blay et al. 2005, 2006; Ribó et al. 2006).

With the period detected here, $4 \mathrm{U} 2206+54$ is the third (if the nature of IGR J16358-4726 as a HMXB is finally confirmed) slowest HMXB pulsar, after 2S 0114+650 and IGR J16358-4726, and the first with a main-sequence companion. 2S $0114+650$ is a 2.78-h pulsar (Finley et al. 1994) accreting matter from a supergiant B1 companion (Reig et al. 1996), while IGR J16358-4726 has been classified as a $\operatorname{sgB}[\mathrm{e}]$
(Chaty et al. 2008, but see Nespoli et al. 2008) with a 1.6-h rotating neutron star (Patel et al. 2004) ${ }^{2}$.

Numerical calculations suggest that there is no significant angular momentum transfer onto the neutron star from the wind of supergiant systems (Ruffert 1999). Even a lower-velocity wind like the one believed to be present in 4U 2206+54 (Ribó et al. 2006) seems to be insufficient to exert a spin-up torque onto the neutron star. Since the transfer of angular momentum during the accretion phase is so inefficient, a spin period of $\sim 5500 \mathrm{~s}$ must have been attained in a previous evolutionary phase. The neutron star's spin evolution in a close binary system (Davies et al. 1979; Davies \& Pringle 1981; Dai et al. 2006) is divided into three phases, of which the accretion phase is the more recent one. The neutron star is born as a millisecond pulsar (pulsar phase) and spin down ocurs by magnetic dipole radiation until $P_{\text {spin }} \sim 1 \mathrm{~s}$. Then the neutron star enters the propeller phase, in which the neutron star spins down because accretion is centrifugally inhibited. This phase continues until the spin period reaches the so-called equilibrium period given by (Li \& van den Heuvel 1999, but see Davies \& Pringle 1981)

$P_{\text {eq }} \approx 20 B_{12}^{6 / 7} \dot{M}_{15}^{-3 / 7} R_{6}^{18 / 7} M_{1.4}^{-5 / 7} \mathrm{~s}$

where $B=10^{12} B_{12} \mathrm{G}$ is the neutron star's dipolar magnetic field strength, $\dot{M}=10^{15} \dot{M}_{15} \mathrm{~g} \mathrm{~s}^{-1}$ is the mass accretion rate, $R=10^{6} R_{6} \mathrm{~cm}$ is the radius of the neutron star, and $M=$ $1.4 M_{1.4} M_{\odot}$ is the mass of the neutron star. Adopting the canonical values $R_{6}=M_{1.4}=1$ and $B_{12}=3.6$ (Blay et al. 2005) and $\dot{M}_{15} \approx L_{\mathrm{X}} R / G M=0.5$ for $L_{\mathrm{X}}=10^{35} \mathrm{erg} \mathrm{s}^{-1}$, the $4 \mathrm{U} 2206+54$ equilibrium period results $P_{\mathrm{eq}} \sim 80 \mathrm{~s}$, far lower than the observed period. We conclude then that the current spin period of 4U 2206+54 cannot be explained in evolutionary terms. A way out is provided by Li \& van den Heuvel (1999), who explain the long spin period of $2 \mathrm{~S} 0114+65$ assuming that the neutron star was born as a magnetar, that is, with $B \gtrsim 10^{14} \mathrm{G}$. Indeed, the propeller effect can spin down the neutron star to $5 \times 10^{3} \mathrm{~s}$ keeping the same values as above but taking $B_{12}$ two orders of magnitude higher.

\subsection{Temporal variability}

The range in X-ray luminosity $(3-30 \mathrm{keV})$ implied from the observations is $0.6-3.1 \times 10^{35} \mathrm{erg} \mathrm{s}^{-1}$, assuming a distance to the source of $2.6 \mathrm{kpc}$ (Blay et al. 2006). That is, 4U 2206+54 displays an amplitude of variability in X-ray flux of about 5. This amplitude is larger than the factor of 2 reported by Ribó et al. (2006) from ASM data, i.e., for X-rays in the 1.3-12 keV band, but consistent with the observations by Corbet \& Peele (2001, see their Fig. 4). Note that the results from the ASM are highly dependent on the minimum flux, which for the ASM is uncertain. In the simplified Bondi-Hoyle accretion model a maximum to minimum orbital X-ray flux ratio of 5 can be explained assuming that the orbital variability is due to changes in the mass accretion rate. In this scenario, an eccentricity of $\sim 0.4$ is needed, somehow higher than that suggested by Ribó et al. (2006).

In addition to the X-ray flux, the power and energy spectral parameters also display variability on time scales of days. If the orbital period of 9.6 days is assumed to be correct, then it seems natural to attribute this variability to the motion of the source through the orbit. Note however, that our results are based on

\footnotetext{
2 The slowest X-ray pulsar is $4 \mathrm{U} 1954+319$ with $P_{\text {spin }}=5 \mathrm{~h}$ (Mattana et al. 2006; Corbet et al. 2008). However, this is a symbiotic X-ray binary with a neutron star component accreting from a low-mass Mtype giant (Masetti et al. 2006b).
} 
Table 4. Results of the exponentially cutoff power law fits to the energy spectra. In the bottom table $N_{\mathrm{H}}$ was kept fixed.

\begin{tabular}{|c|c|c|c|c|c|c|c|c|}
\hline Bin & Orbital phase ${ }^{a}$ & $N_{\mathrm{H}}^{b}$ & $E_{\text {cut }}$ & $E_{\text {fold }}$ & $\Gamma$ & Norm & $F_{3-30}^{(c)}$ & $\chi_{v}^{2}$ (d.o.f.) \\
\hline A & $0.87 / 0.93$ & $1.54_{-0.83}^{+0.89}$ & $6.34_{-0.51}^{+0.68}$ & $13.34_{-1.95}^{+2,49}$ & $1.29_{-0.16}^{+0.15}$ & $0.028_{-0.028}^{+0.009}$ & 3.33 & $0.65(51)$ \\
\hline B & $0.06 / 0.03$ & $1.54_{-0.58}^{+0.59}$ & $6.32_{-0.38}^{+0.47}$ & $14.46_{-1.60}^{+1.93}$ & $1.32_{-0.11}^{+0.10}$ & $0.032_{-0.006}^{+0.007}$ & 3.67 & $0.72(51)$ \\
\hline $\mathrm{C}$ & $0.17 / 0.09$ & $0.63_{-0.63}^{+1.01}$ & $6.23_{-0.43}^{+0.60}$ & $12.03_{-1.55}^{+2.58}$ & $1.28_{-0.13}^{+0.18}$ & $0.012_{-0.002}^{+0.004}$ & 1.32 & $0.72(51)$ \\
\hline D & $0.33 / 0.17$ & $0.94_{-0.94}^{+1.01}$ & $6.13_{-0.51}^{+0.63}$ & $11.99_{-2.20}^{+2.78}$ & $1.44_{-0.20}^{+0.18}$ & $0.009_{-0.003}^{+0.004}$ & 0.76 & $1.37(51)$ \\
\hline $\mathrm{E}$ & $0.50 / 0.25$ & $0.95_{-0.95}^{+1.11}$ & $6.24_{-0.63}^{+0.45}$ & $12.24_{-2.01}^{+3.20}$ & $1.44_{-0.17}^{+0.18}$ & $0.010_{-0.003}^{+0.004}$ & 0.82 & $0.63(51)$ \\
\hline A & $0.87 / 0.93$ & 0.9 & $6.00_{-0.27}^{+0.26}$ & $11.88_{-0.57}^{+0.61}$ & $1.17_{-0.04}^{+0.04}$ & $0.023_{-0.001}^{+0.001}$ & 3.26 & $0.67(52)$ \\
\hline B & $0.06 / 0.03$ & 0.9 & $5.99_{-0.21}^{+0.22}$ & $12.83_{-0.53}^{+0.57}$ & $1.21_{-0.03}^{+0.03}$ & $0.026_{-0.001}^{+0.001}$ & 3.60 & $0.77(52)$ \\
\hline $\mathrm{C}$ & $0.17 / 0.09$ & 0.9 & $6.34_{-0.33}^{+0.33}$ & $12.62_{-0.82}^{+0.93}$ & $1.33_{-0.05}^{+0.04}$ & $0.013_{-0.001}^{+0.001}$ & 1.33 & $0.63(52)$ \\
\hline D & $0.33 / 0.17$ & 0.9 & $6.12_{-0.32}^{+0.33}$ & $11.87_{-0.82}^{+0.94}$ & $1.43_{-0.05}^{+0.04}$ & $0.009_{-0.001}^{+0.001}$ & 0.76 & $0.71(52)$ \\
\hline $\mathrm{E}$ & $0.50 / 0.25$ & 0.9 & $6.25_{-0.39}^{+0.33}$ & $12.17_{-0.96}^{+1.09}$ & $1.44_{-0.06}^{+0.04}$ & $0.009_{-0.001}^{+0.002}$ & 0.82 & $1.33(52)$ \\
\hline
\end{tabular}

${ }^{a}$ At mid-point for $P_{\text {orb }}=9.56 \mathrm{~d}$ and $2 P_{\text {orb }}=19.12 \mathrm{~d}$, respectively; ${ }^{b}$ hydrogen column density in units of $10^{22} \mathrm{~cm}^{-2} ;{ }^{c}$ unabsorbed flux in units of $10^{-10} \mathrm{erg} \mathrm{s}^{-1} \mathrm{~cm}^{-2}$.

less than one orbit. The power spectra of 4U 2206+54 are well described by a single power law, whose index and rms amplitude decrease as the count rate decreases (Table 2). There is no sign of a break at low frequencies (flat-topped noise) in the frequency range covered by the power spectra. Red noise dominates the power spectrum down to frequencies as low as $1 \mathrm{mHz}$. The decrease in the amplitude of the X-ray variability (rms) with decreasing X-ray flux seems to indicate a large scale change in the structure of the wind. One possible explanation is that the wind was highly inhomogeneous during intervals $\mathrm{A}, \mathrm{B}$ and $\mathrm{C}$, during periastron passage, as usually observed in systems with supergiant companions, and became smoother further out, in intervals $\mathrm{C}$ and $\mathrm{D}$. Assuming a mass of $18 M_{\odot}$ for the $\mathrm{O} 9.5 \mathrm{~V}$ primary and the canonical neutron star mass of $1.4 M_{\odot}$ for the accretor, the change in the wind structure must have occured in between intervals $\mathrm{C}$ and $\mathrm{D}$, which in terms of radial distance corresponds to $\sim 5.7 R_{*}$ for $P_{1}=9.6 \mathrm{~d}$ and $\sim 6.95 R_{*}$ for $P_{2}=19.25 \mathrm{~d}$. The detection of X-ray emission in intervals D and E in 4U 2206+54 contrasts with what is observed in classical supergiant systems for which the X-ray emission is hardly detected when the compact object is located beyond $3 R_{*}$ (Negueruela et al. 2008; Blay et al. 2008).

The 3-30 keV spectrum of $4 \mathrm{U} 2206+54$ can be described with a power law with $\Gamma=1.5$ and a high-energy cutoff with $E_{\text {cut }}=6.7 \mathrm{keV}$ (see Sect. 4.1). The power-law index increases as the X-ray flux decreases (Table 4). The high-flux intervals (A and $\mathrm{B}$ ) show a harder spectrum than the low-flux states $(\mathrm{C}, \mathrm{D}$ and E). This behaviour is the opposite to that found in other $\mathrm{X}$-ray sources. The explanation could be, again, in the low X-ray brightness of this source. Within the framework of bulk motion Comptonization, that is, assuming that Comptonization occurs in the accretion flow near the neutron star surface (accretion column), the surface of the neutron star acts as source of feedback against the infalling material: when the accretion increases, so does the X-ray flux. The ram pressure exerted by the X-ray source tends to hamper accretion and therefore complicates the Comptonization process. In the very bright X-ray sources, the feedback decreases the efficiency of Comptonization and produces a high-soft low-hard behaviour. In 4U 2206+54 however, with its main sequence donor, the X-ray source is never pushed to the limit where the X-rays can hamper the accretion process. With no feedback from the surface of the neutron star, the source becomes harder when accreting material becomes available, presumably at the periastron passage.
One of the controversial results of $4 \mathrm{U} 2206+54$ in the X-ray band is the shape of the hardness-intensity diagram. Negueruela \& Reig (2001) found a correlation between the X-ray colours and the X-ray intensity, in that the X-ray spectrum becomes harder as the flux increases. Figure 3 confirms these results. This figure shows the hardness-intensity diagram for three different ratios. In all three cases the change in intensity is approximately the same, namely, a factor of 10. In contrast, Masetti et al. (2004) did not find any variability of the hardness ratio with intensity in a BeppoSAX observation on 1998 November. Masetti et al. (2004) suggested that the hardness-intensity dependence might become evident at higher luminosities, as the X-ray luminosity during the Negueruela \& Reig (2001) observations was about one order of magnitude higher than that of Masetti et al. (2004). To investigate this possibility we obtained hardness-intensity diagrams for interval $\mathrm{B}$ and $\mathrm{D}$, that is, when the $\mathrm{X}$-ray flux was maximum and minimum, respectively. We define the hardness ratios as $H R 1=7-10 \mathrm{keV} / 4-7 \mathrm{keV}$ and $H R 2=10-15 \mathrm{keV} / 4-$ $7 \mathrm{keV}$. Fitting $H R 1$ and $H R 2$ as a function of $4-30 \mathrm{keV}$ intensity to a constant does not give acceptable fits for interval $\mathrm{B}$. The fits to interval D are acceptable (in the sense $\chi_{\mathrm{r}}^{2}<2$ ). However, the reason for this acceptable fit is because the error bars are larger. As an example, a linear fit $(y=a x+b)$ to the HR2-intensity diagram of interval D reduces the $\chi^{2}$ from 419 (259 d.o.f.) to 290 (258 d.o.f.). An F-distribution test gives a chance improvement of only $0.18 \%$. That is, the addition of a linear component is significant above a 3- $\sigma$ level. In fact, irrespective of the X-ray flux, as the intensity increases by a factor 10 , the hardness ratios $H R 1$ and $H R 2$ increase by $\sim 25 \%$. In summary, the hardness-intensity dependence becomes more evident at higher source luminosities (as Masetti et al. 2004, had suggested). However, this effect is most likely due to low statistics rather than the disappearance of the correlation.

\subsection{Soft component}

When a broader energy range is considered, the high-energy cutoff power-law model does not give acceptable fits and a soft excess at energies below $2 \mathrm{keV}$ shows up in the residuals. More physical models involve Comptonization. Either thermal or bulk-motion Comptonization produce good fits to the X-ray energy continuum in the $3-80 \mathrm{keV}$ band. In either case a blackbody component with $k T_{\mathrm{bb}}=0.5 \mathrm{keV}$ is required to account for the low-energy photons. 
The presence of a blackbody component supports the suggestion by Hickox et al. (2004) that soft excess is common (probably ubiquitous) in X-ray pulsars. For high luminosity $\left(L_{\mathrm{X}} \sim\right.$ $10^{38} \mathrm{erg} \mathrm{s}^{-1}$ ) pulsars, the soft component stems from reprocessing of hard X-rays from the neutron star in the inner edge of an accretion disc. In less luminous systems, other mechanisms, such as emission from diffuse gas or from the neutron star surface, can be at work. To get a rough estimate of the size of the soft radiation emission site, we compute the radius of the circular blackbody spot that would produce the observed soft luminosity, according to data in Table 3 . We get a radius of $R_{\mathrm{bb}} \sim 1.5 \mathrm{~km}$. This value is too small to come from the entire surface of the neutron star, a hot corona, or an accretion disc. The lack of a disc in 4U 2206+54 was discussed in Torrejón et al. (2004). This size is of the same order as that deduced previously from the temperature of the seed soft photons $R_{\mathrm{W}} \approx 1.5 \mathrm{~km}$. Then we tried to tie the temperature of both components during the fits. The resulting statistics were significantly worse. It seems, therefore, that the spectrum comes from two sites, with the same characteristic size but different temperature. They could reflect a gradient in temperature from the hot spot (of radius $R_{\mathrm{W}}$ in the base of the accretion column) and the surface surrounding the spot (just outside the column) and into the relatively cold surface.

When the soft excess is modelled with a blackbody component, the blackbody spectral parameters (temperature and emission radius) strongly depend on the source luminosity, which in turn, depends on the accretion mechanism. In Fig. 9, we plot the temperature and radius of the emitting region allegedly causing the soft excess. High-luminosity systems display lower temperatures and larger radii (open squares). In high-luminosity pulsars, such as SMC X-1, Cen X-3 and LMC X-4, accretion occurs via Roche lobe overflow and an accretion disc is formed. In these systems $k T=0.1 \mathrm{keV}$ and $R_{\mathrm{bb}}=100 \mathrm{~km}$, typically (see e.g. Table 5, in Hickox et al. 2004), and the soft excess is believed to be due to reprocessing of hard X-rays by optically thick accreting material. In contrast, low-luminosity systems (filled circles), such as X Per (Coburn et al. 2001), A 0535+26 (Mukherjee \& Paul 2005) and RX J0146.9+6121 (La Palombara \& Mereghetti 2006) have $k T=1.2 \mathrm{keV}$ and $R_{\mathrm{bb}}=0.1 \mathrm{~km}$. In these systems the neutron star captures material from the high-density wind from the circumstellar disc of the Be companion. The origin of the soft excess would be (part of) the neutron star surface. Note also that the high-luminosity pulsars mentioned above contain supergiant companions, while the low-luminosity systems harbour main-sequence stars. In between these extreme cases one finds the wind-fed supergiant systems (filled triangles), such as Vela X-1, with $k T_{\mathrm{bb}}=0.2 \mathrm{keV}$ and $R_{\mathrm{bb}}=70 \mathrm{~km}$ (Hickox et al. 2004 ) and $2 \mathrm{~S} 0114+650$, with $k T_{\mathrm{bb}}=0.3 \mathrm{keV}$ and $R_{\mathrm{bb}}=200 \mathrm{~km}$ (Masetti et al. 2006a). In these systems the thermal component responsible for the soft excess is likely to be a cloud of diffuse plasma around the neutron star (Hickox et al. 2004).

The star-like symbols in Fig. 9 represent three different observations of 4U 2206+54 (Masetti et al. 2004; Torrejón et al. 2004, and present work). 4U 2206+54 appears close to the $\mathrm{Be} / \mathrm{X}$-ray binaries, as expected if the soft excess arises from a hot spot on the neutron star surface. It is not clear how such a hot spot can be formed in 4U 2206+54 as accretion is also driven through a stellar wind (Ribó et al. 2006). Perhaps the much slower (and presumable denser) wind in 4U2206+54 might make the accretion configuration be more similar to that of persistent Be/X-ray binaries.

The open circle corresponds to the peculiar Be/X-ray binary SAX J2103.5+4545 (Inam et al. 2004). This source is similar to $4 \mathrm{U} 2206+54$ in that the spin and orbital periods are

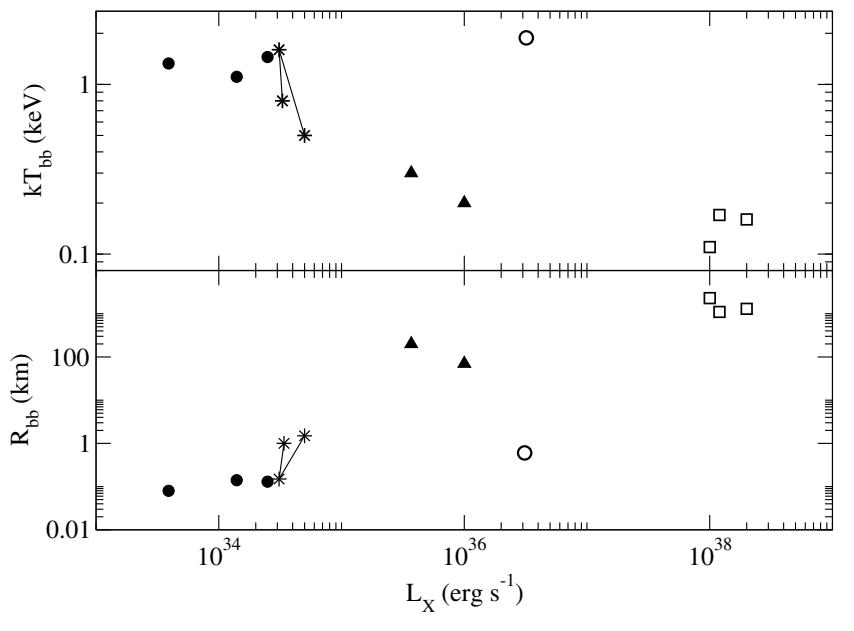

Fig. 9. Variation of the characteristic temperature (upper panel) and radius (lower panel) with X-ray luminosity of the emitting region producing the soft excess, if modelled with a blackbody. Squares represent high-luminosity disc-fed supergiants (Cen X-3, SMC X-1 and LMC X-4); filled circles are low-luminosity persistent Be/X-ray binaries (X-Per, A $0535+262$ and LS I +61 235); triangles correspond to the wind-fed supergiants (Vela X-1 and 2S 0114+65); the open circle is SAX J2103.5+4545; stars mark the position of 4U 2206+54 for three different observations (see text for details).

typical of supergiant X-ray binaries, but the primary component of the binary is a main-sequence star. In both systems the orbits have $e=0.4$. The X-ray variability is, however, rather different. SAX J2103.5+4545 presents X-ray bright states that last for a few months and extended (few years) X-ray faint states. During the bright states an accretion disc is formed around the neutron star (Baykal et al. 2002) and a decretion circumstellar disc is formed around the Be star (Reig et al. 2004). These discs are probably short lived and appear during the high X-ray emission states only. Reig et al. (2005) found that SAX J2103.5+4545 was emitting X-rays even after the complete loss of the circumstellar disc. In this state, Reig et al. (2005) argue that the X-rays are the result of wind-fed accretion, that is, as in $4 \mathrm{U} 2206+54$.

The position of these two sources in the $P_{\text {spin }}-P_{\text {orb }}$ diagram (Fig. 10) reinforces their similarity. They fall in the wind-fed supergiant region. Since this diagram reflects the type of massloss/accretion mechanism (Corbet 1986), it is not surprising to find 4 U 2206+54 and SAX J2103.5+4545 in this region. What makes them peculiar is that they both contain a main-sequence donor.

The blackbody temperature and radius (scaled to a distance of $6.5 \mathrm{kpc}$; Reig et al. 2004) of SAX J2103.5+454 shown in Fig. 9 (open circle) were obtained during a bright state (Inam et al. 2004), which may explain the isolated position in the plot. Note that the value of the radius and blackbody temperature are consistent with those of the low-luminosity $\mathrm{Be} / \mathrm{X}$-ray binaries (filled circles). The difference appears in the X-ray luminosity. The higher luminosity in SAX J2103.5+454 can be explained if an accretion disc was present during the observations. The detection of a quasi-periodic oscillation at $0.044 \mathrm{~Hz}$ (Inam et al. 2004) and the correlation between spin-up/down with X-ray flux (Baykal et al. 2007) indeed supports the presence of an accretion disc. 


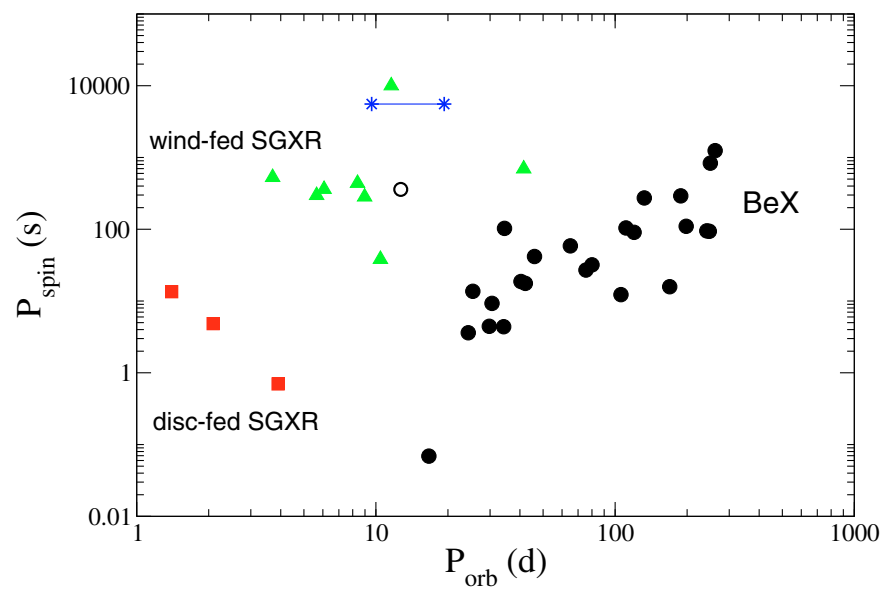

Fig. 10. $P_{\text {spin }}-P_{\text {orb }}$ diagram. The two joined star-like symbols represent $4 \mathrm{U} 2206+54$ for the two suggested orbital periods. The open circle corresponds to SAX J2103.5+4545.

\section{Conclusion}

We have finally unveiled the nature of the compact companion in the high-mass X-ray binary $4 \mathrm{U} 2206+54$. The RXTE light curve of 4U $2206+54$ appears to be modulated with a period of $\sim 1.54 \mathrm{~h}$ that we interpret as the spin period of the neutron star. Evidence for this modulation is also found in INTEGRAL and EXOSAT data. Thus we rule out that the periodicity is due to the $R X T E$ orbit. $4 \mathrm{U} 2206+54$ becomes the slowest rotating neutron star in a high-mass X-ray pulsar with a main-sequence companion. The X-ray flux and the spectral index of the power-law components that fit energy and power spectra exhibit variability on time scales of days. The amplitude of variability of the X-ray flux changed by a factor of 5 on time scales of days. The powerlaw index is larger (i.e. steeper spectrum) during low-flux states in the energy spectra and during high-flux states in the power spectra. We attribute this variability to changes in the mass accretion rate as the neutron star orbits the O-type companion in a moderately eccentric orbit. The time span covered by the observations is shorter than any of the two suggested orbital periods, hence further monitoring campaigns covering a larger fraction of the orbit are needed to pin down the correct orbital period. However, the fact that the maximum of the PCA light curve occurs at phase zero, i.e., in coincidence with the ASM maximum when folded onto the 9.6-day period and the detection of a strong signal at $\sim 815000 \mathrm{~s}$ in the RXTE periodogram would favour the shorter period. The detection of a soft component in the energy spectrum has been interpreted as emission from the polar caps. The plot of the blackbody emitting radius and temperature as a function of X-ray luminosity constitutes a useful tool to distinguish the type of accretion mechanism at work in the X-ray binaries that show a soft excess.

Acknowledgements. This research was supported by the European Union Marie Curie grant MTKD-CT-2006-039965. This research is supported by the DGI of the Spanish Ministerio de Educación y Ciencia under grants AYA2005-00095 and AYA2007-68034-C03-01 and FEDER funds. J.M.T. acknowledges the support of the Spanish Ministerio de Educación y Ciencia under grant PR2007-0176. This research has made use of data obtained through the INTEGRAL Science Data Center (ISDC), Versoix, Switzerland.

\section{References}

Abubekerov, M. K. 2004, Astron. Rep., 48, 649

Baykal, A., Stark, M. J., \& Swank, J. H. 2002, ApJ, 569, 903 Baykal, A., Inam, S. Ç., Stark, M. J., et al. 2007, MNRAS, 374, 1108 Belloni, T., \& Hasinger, G. 1990, 230, 103

Blay, P., Ribó, M., Negueruela, I., et al. 2005, A\&A, 438, 963 Blay, P., Negueruela, I., Reig, P., et al. 2006, A\&A, 446, 1095

Blay, P., Martínez-Núñez, S., Negueruela, I., et al. 2008, A\&A, 489, 669 Casares, J., Ribas, I., Paredes, J. M., Martí, J., \& Allende Prieto, C. 2005a, MNRAS, 360, 1105

Casares, J., Ribó, M., Ribas, I., et al. 2005b, MNRAS, 364, 899 Chaty, S., Rahoui, F., Foellmi, C., et al. 2008, A\&A, 484, 783

Clark, J. S., Goodwin, S. P., Crowther, P. A., et al. 2002, A\&A, 392, 909 Coburn, W., Heindl, W. A., Gruber, D. E., et al. 2001, ApJ, 552, 738 Corbet, R. H. D. 1986, MNRAS, 220, 1047

Corbet, R. H. D., \& Peele, A. G. 2001, ApJ, 562, 936

Corbet, R. H. D., Markwardt, C. B., \& Tueller, J. 2007, ApJ, 655, 458 Corbet, R. H. D., Sokoloski, J. L., Mukai, K., et al. 2008, ApJ, 675, 1424

Courvoisier, T. J.-L., Walter, R., Beckmann, V., et al. 2003, A\&A, 411, L53 Dai, H.-L., Liu, X.-W., \& Li, X.-D. 2006, ApJ, 653, 1410

Davies, R. E., \& Pringle, J. E. 1981, MNRAS, 196, 209

Davies, R. E., Fabian, A. C., \& Pringle, J. E. 1979, MNRAS, 186, 779

Dhawan, V., Mioduszewski, A., \& Rupen, M., 2006, in Proceedings of the

VI Microquasar Workshop: Microquasars and Beyond, September 18-22, Como, Italy, 52

Finley, J. P., Taylor, M., \& Belloni, T. 1994, ApJ, 429, 356

Gros, A., Goldwurm, A., Cadolle-Bel, M. et al. 2003, A\&A, 411, L179

Hickox, R. C., Narayan, R., \& Kallman, T. R. 2004, ApJ, 614, 881

Inam, S. Ç, Baykal, A., Swank, J. \& Stark, M. J. 2004, ApJ, 616, 463

Jahoda, K., Swank, J. H., Stark, M. J., et al. 1996, EUV, X-ray and Gamma-ray Instrumentation for Space Astronomy VII, ed. O. H. W. Siegmund, \& M. A. Gummin, SPIE 2808, 59

La Palombara, N., \& Mereghetti, S. 2006, A\&A, 455, 283

Lenz, P., \& Breger, M. 2005, CoAst, 146, 53

Levine, A. M., Bradt, H., Cui, W., et al. 1996, ApJ, 469, L33

Li, X.-D., \& van den Heuvel, E. P. J. 1999, ApJ, 513, L45

Masetti, N., Dal Fiume, D., Amati, L., et al. 2004, A\&A, 423, 311

Masetti, N., Orlandini, M., Dal Fiume, D., et al. 2006a, A\&A, 445, 653

Masetti, N., Bassani, L., Bazzano, A., et al. 2006b, A\&A, 453, 295

Mattana, F., Götz, D., Falanga, M. et al. 2006, A\&A, 460, 1

Miyamoto, S., Kimura, K., Kitamoto, S., Dotani, T., \& Ebisawa, K. 1991, ApJ, 383, 784

Mukherjee, U., \& Paul, B. 2005, A\&A, 431, 667

Negueruela, I., \& Reig, P. 2001, A\&A, 371, 1056

Negueruela, I., Torrejón, J. M., Reig, P., Ribó, M., \& Smith, D. M. 2008, in AIP Conf. Proc., 1010, 252 [arXiv: 0801.3863]

Nespoli, E., Fabregat, J., \& Mennickent, R. E. 2008, ATel 1450

Patel, S. K., Kouveliotou, C., Tennant, A., et al. 2004, ApJ, 602, L45

Reig, P., Chakrabarty, D., Coe, M. J., et al. 1996, A\&A, 311, 879

Reig, P., Negueruela, I., Fabregat, J., et al. 2004, A\&A, 421, 673

Reig, P., Negueruela, I., Papamastorakis, G., Manousakis, A., \& Kougentakis, T. 2005, A\&A, 440, 637

Ribó, M., Negueruela, I., Blay, P., Torrejón, J. M., \& Reig, P. 2006, A\&A, 449, 687

Ribó, M., Paredes, J. M., Moldón, J., Martí, J., \& Massi, M. 2008, A\&A, 481, 17

Roberts, D. H., Lehár, J., \& Dreher, J. W. 1987, AJ, 93, 968

Rothschild, R. E., Blanco, P. R., Gruber, D. E., et al. 1998, ApJ, 496, 538

Ruffert, M. 1999, A\&A, 346, 861

Saraswat, P., \& Apparao, K. M. V. 1992, ApJ, 401, 678

Steiner, J. E., Ferrara, A., Garcia, M., et al. 1984, ApJ, 280, 688

Stellingwerf, R. F. 1978, ApJ, 224, 953

Torrejón, J. M., Kreykenbohm, I., Orr, A., Titarchuk, L., \& Negueruela, I. 2004, A\&A, 423, 301

Turner, M. J. L., Smith, A., \& Zimmermann, H. U. 1981, SSRv, 30, 513

Ubertini, P., Lebrun, F., Di Cocco, G., et al. 2003, A\&A, 411, L131 